\title{
UN COLLAGE DE FUENTES EN LA HISTORIA DE MARCO ANTONIO Y CLEOPATRA (1639) DE CASTILLO SOLÓRZANO
}

\author{
JONATHAN BRADBURY \\ University of Exeter, Reino Unido \\ j.bradbury@exeter.ac.uk
}

$\mathrm{E}$

1 Siglo de Oro no produjo ninguna representación brillante y duradera de Cleopatra; sin embargo, la última reina del antiguo Egipto mantuvo una presencia discreta en la literatura del tiempo de los Austrias. Asoma por el teatro en el Marco Antonio y Cleopatra (1582) de Diego López de Castro ${ }^{1}$, y en Los áspides de Cleopatra de Francisco de Rojas Zorrilla, pieza que se estrenó a principios de los años cuarenta de la siguiente centuria ${ }^{2}$. Entre ambas fechas, se registra una alusión a una comedia titulada Marco Antonio y Cleopatra dentro del repertorio de la compañía de Juan de Acacio en 1627 (González Cañal 2008: 273), y cabe suponer que Cleopatra desempeñaba un papel importante en Los triunfos de Octaviano de Lope de Vega, comedia escrita -al decir del mismo Fénix- antes de 1604 y ahora perdida ${ }^{3}$.

El tratamiento poético más sustancial de las vicisitudes de la soberana egipcia se cifra en el Canto de Antonio y Cleopatra de Francisco de Borja, publicado en 1640 e incluido en sus Obras en verso (Borja 1648: 110-145); casi un siglo antes, se había publicado la segunda interpretación digna de nota -por lo que atañe al uso del verso-: Cleopatra desfila por dos composiciones del Libro de los cuarenta cantos (1550) de Alonso de Fuentes, ambas acompañadas de glosas moralizadoras en prosa (Fuentes 1587: ff. 90v-104v; ff. 288v-302r). Es verdad que contamos

\footnotetext{
Véase Rennert (1908) para una breve descripción y la transcripción entera de la comedia.

2 Sobre la comedia de Rojas Zorrilla, publicada en la Segunda parte de sus dramas en 1645, véase González Cañal (2008), quien habla también de las dos representaciones dramáticas subsecuentes a la de Rojas durante el Seiscientos: Marco Antonio y Cleopatra, sin autor ni fecha, y Los tres señores del mundo, de Luis Belmonte Bermúdez, publicada en 1653.

3 El título comparece en la lista redactada por el mismo Lope en 1604 en El peregrino en su patria (Vega 1973: 60)
}

Edad de Oro, XXXVI (2017), pp. 93-107, ISSN: 0212-042 - DOI http://dx.doi.org/10.15366/edadoro2017.36.006 
asimismo con breves poemas, verbigracia el soneto de Lope «Cleopatra a Antonio en oloroso vino», compilado en las Rimas de 1604, relectura del episodio fantástico y consabido de la perla disuelta en vinagre, a zaga del relato de la Historia Natural (9.119-121) de Plinio.

La misma escena se documenta en obras misceláneas o enciclopédicas del Renacimiento (Sainz de la Maza 2009): desde la Silva de varia lección (1540) de Pedro Mexía (2003: 620-621) y sus Diálogos o Coloquios (1547) (Mexía 2004: 300301), a la traducción llevada a cabo en 1586 por Andrea Pescioni de las Histoires prodigieuses (1560) de Pierre Boaistuau (1586: 90v) y los Diálogos familiares de la agricultura cristiana (1589) de Juan de Pineda (1963: 220-221). Si Boaistuau sitúa el hecho maravilloso en el contexto de los suntuosos banquetes que celebró la hija de Ptolomeo XII para recibir a Marco Antonio, y persigue sobre todo la admiratio, los interlocutores del coloquio de Pineda lo juzgan bajo la luz de la escandalosa conducta de la reina, tomando como piedra de toque la Monarquía eclesiástica (1576) del mismo Pineda. En dicho volumen, Cleopatra es el sujeto de dos capítulos (36 y 37, al final del libro IX) (Pineda 1588: ff. 364v-369r) donde se la define con trazos relativos a su inmoralidad, lascivia y avidez, mayores si cabe que las que había lucido en el retrato de la Historia imperial y cesárea (1545) de Mexía, quien reservó a Cleopatra una presencia relevante en las secciones dedicadas a Julio César y César Augusto (Mexía 1655: 1-34)

Si las obras de Mexía y Pineda constituyen un par de ejemplos capitales de la historiografía vernácula en el Renacimiento español, el asedio más reseñable durante el Barroco, la Historia de Marco Antonio y Cleopatra de Alonso de Castillo Solórzano, es un texto del todo distinto. Se publicó en Zaragoza en 1639, al final de una década que testimonia la aparición de los libros más ambiciosos y variados del autor vallisoletano ${ }^{5}$. El hecho de que las aprobaciones y la suma del privilegio se firmaran en la capital aragonesa durante el mes de octubre de 1635 da fe de que el texto se había ultimado cuatro años antes de su salida al mercado. Disponemos además de noticias fidedignas acerca de una versión primitiva, ya lista para la imprenta en 1625: las aprobaciones de Juan de Jáuregui y Francisco Boil en los preliminares de Tiempo de regocijo, entregado a los tórculos en Madrid en 1627, se rubricaron en la misma ciudad en 1625, y aluden respectivamente a una «Vida de Cleopatra» y a una «Historia de Cleopatra» (Castillo Solórzano 1907: 184 y 185$)^{6}$.

No consta que aquella versión viese la luz, y se antoja razonable inferir que el manuscrito alcanzó su forma definitiva en 1639. Podemos suponer, además, que

\footnotetext{
Sobre la representación de Cleopatra en las dos obras, véase Jiménez Belmonte (2011a: 287-305). Sobre la vida y las obras de Castillo, véase Bonilla Cerezo (2012).

En las últimas líneas del libro, Castillo promete «dar a la estampa a La reina Cleopatra» (435), siempre que la presente obra mereciera una acogida positiva.
} 
el proyecto de 1625 no contenía el elemento más llamativo de la obra definitiva; es decir, la mezcla de prosa y verso: veintisiete poemas en total, salidos de la pluma de Castillo y otros personajes mayores y menores de los cenáculos de aquel tiempo. El vallisoletano llama la atención sobre este detalle en su breve prefacio, donde subraya la calidad de «los grandes poetas que le honran [este volumen] con sus elegantes versos» (Castillo Solórzano 1639: «Prólogo»); y nos parece probable que la novedad de esta mixtura de poesía y prosa en la historiografía, junto a la presencia de autores como Lope de Vega y Pérez de Montalbán, hubieran sido encomiadas por los aprobadores - como hizo Diego Amigo en 1635- si los poemas hubieran formado parte del texto temprano ${ }^{7}$.

Dichos poemas representan la faceta más evidente de lo que podemos denominar el collage de la Historia de Marco Antonio y Cleopatra y se interpolan irregularmente desde el segundo capítulo al decimocuarto (y último). Si bien es cierto que Castillo destaca por ser uno de los exponentes más notorios de la literatura mixta - específicamente la prosa intercalada con versos-a lo largo del Barroco español, la Historia de Marco Antonio y Cleopatra se levanta como una rareza entre sus obras, ya que la mayoría de los poemas son ajenos ${ }^{8}$. Un total de doce ingenios -más Castillo- contribuyeron con sus versos al libro que nos ocupa: Gaspar Mercader, Juan Pérez de Montalbán, Francisco Tamayo y Porres, Jacinto Navarro, Vicente Gascón de Siurana, José de Valdivielso, Monserrat de Cruyllas, Luis de Villanova y Sancho de Molina y Soto, le brindaron una composición; Francisco Novella y Lope de Vega regalaron un par de ellas; y tres Francisco Diego de Sayas, lo que lo convierte en el autor más prolífico del volumen, excepción hecha del propio Castillo, que incluyó once de su puño y letra. De estos veintisiete poemas, dieciséis giran en torno a Marco Antonio y/o Cleopatra, siete se centran en Pompeyo y Julio César, y tres exponen las hazañas de Octaviano, más tarde César Augusto. La segunda composición de Novella es un poema más que genérico sobre la lisonja, sin ninguna deuda con los personajes de la Antigüedad romana. Casi todos son sonetos, si bien encontramos dos piezas en octavas: la de Gascón de Siurana y el primer texto de Novella.

Podemos con buenos motivos poner en cuarentena el título de «grandes poetas» que atribuyó Castillo a sus cómplices; aunque es verdad que un buen número de las poesías son de calidad, solo a Lope, Valdivielso, Pérez de Montalbán y quizá Mercader se los pueden estimar como figuras literarias establecidas, mientras que los otros contribuidores son hábiles diletantes, ahora olvidados, cuya poesía surgió en gran parte de las academias o certámenes. El ámbito literario más

\footnotetext{
$7 \quad$ Recuérdese que el mismo Jáuregui había publicado ya un soneto sobre la derrota de Marco Antonio en la Batalla de Accio y la influencia de Cleopatra en tamaño fracaso: «Sobre las ondas acosado Antonio», la primera composición en sus Rimas después de la Aminta (Jáuregui 1618: 93).

8 Sobre Castillo como autor de obras mixtas, véase Arredondo (2006).
} 
representado en la poesía de la Historia es el de Valencia, de donde provienen siete de los colaboradores. Esta clave geográfica refleja la importancia de la urbe levantina en la trayectoria de Castillo a lo largo de la tercera y cuarta décadas del siglo XVII, pues acompañó en calidad de maestresala al virrey Luis Fajardo de Requeséns (1628-1631) y después a su hijo Pedro Fajardo y Zúñiga, que lo relevaría en el cargo (1631-1635). Luego es probable que Castillo conociera a algunos de los ingenios valencianos en las academias a orillas del Turia ${ }^{9}$. De los cinco poetas sin vínculos con Valencia, cuatro tienen que ver con Madrid, mientras que Sayas no solo es el colaborador más activo de Castillo en esta obra, sino también el único aragonés, circunstancia atribuible al poco tiempo de asiento en Zaragoza cuando Castillo sometió su Historia al criterio de los censores. Tiene razón, por tanto, Jiménez Belmonte (2011b: 321) al caracterizar este libro como «una suerte de academia virtual» que entreteje tres contextos urbanos importantes (Valencia, Madrid, Zaragoza); igual que en las academias del mundo real, los diletantes, tanto del entorno universitario como de la nobleza, interactúan con autores célebres.

La falta de certeza en cuanto al método o a los métodos que informaron la adquisición por parte de Castillo de las poesías ajenas es un problema común a todas las composiciones, con la sola excepción del primer soneto de Lope, «Cleopatra a Antonio en oloroso vino», que ya había aparecido en letras de molde y se incorporó muy torpemente al nuevo contexto. Si el soneto sobre la disolución de la perla se presta fácilmente a la narración de los banquetes ofrecidos por Cleopatra, Castillo no se cuida lo bastante de eliminar el apóstrofe final al recipiente: «Quedó la perla sola en testimonio / de que no tuvo igual hasta aquel día, / bella Lucinda, que naciste al mundo» (Castillo Solórzano 1639: 54). Por lo que respecta a los demás poemas, no sabemos cuáles circulaban ya entonces, ni en qué forma; y tampoco si alguno de ellos es producto de una solicitud directa de Castillo. Así las cosas, no podemos precisar el grado de especificidad que acompañara a la hipotética petición.

Castillo se relacionaba con el círculo de Lope -al que escudaban Valdivielso y Pérez de Montalbán- en el Madrid en los años '20 del Seiscientos, y hemos documentado sus tratos con otros poetas de los que se dan cita en la Historia en los paratextos de obras anteriores del vallisoletano: Navarro, Gascón de Siurana, Cruyllas y Tamayo y Porres habían contribuido ya con décimas a los preliminares

9 Mas i Usó (1999) ofrece detalles sobre cuatro de los valencianos cuyas poesías se suman a la Historia de Marco Antonio y Cleopatra: Gascón de Siurana (450-451), Mercader (471-472), Navarro (479) y Novella (480). No obstante, la hipótesis avanzada por King (1963: 127-128), la de una sesión académica dedicada al tema de Cleopatra durante la estancia de Castillo en Valencia, que habría luego informado su Historia, se ve cuestionada por el hecho de que solo cuatro de los siete escritores levantinos que donaron poesías al volumen escribieron a propósito de la reina egipcia. Además, todas estas composiciones resumen los últimos episodios de la historia, desde la Batalla de Accio hasta la muerte de Cleopatra. 
del Lisardo enamorado, publicado en Valencia en 1629, y otra décima de Tamayo y Porres se registra entre los materiales que preceden a la Huerta de Valencia, publicada en idénticos lugar y año. Asimismo, la primera novela de las Noches de placer (1631), «Las dos dichas sin pensar», se dedica a Mercader, el más distinguido de los académicos valencianos; mientras que la tercera, «La ingratitud y el castigo», va enderezada a Cruyllas. El poeta con los lazos más estrechos con la carrera de Castillo hasta esa fecha es Molina y Soto, que se había sumado a los preliminares de la Huerta de Valencia, los Escarmientos de amor moralizados (1628) y el Patrón de Alcira (1636). No en balde, en el año 1634 hallamos también un romance suyo, «Cuando los hados se indignan», en la colectánea titulada Fiestas del jardín (Castillo Solórzano 1634: 147-151). Por último, Castillo, como antesala de una de sus comedias, incrustó una composición de Villanova, escrita con el mismo metro, «Oh, qué linda sales, niña», en el último "divertimiento" de la Huerta de Valencia (Castillo Solórzano 1944: 224-225).

Con todo, no hay duda de que trabó contacto previo con la mayoría de sus coagentes en la Historia, aun cuando la participación del aragonés Sayas siga envuelta en tinieblas, toda vez que no consta ninguna amistad entre él y Castillo. Eso sí, el vallisoletano se afanó en otorgar una posición de privilegio dentro de su volumen a un intelectual aragonés muy respetado, potenciando así la campaña de autopromoción que había desplegado desde que se mudara a Zaragoza durante la primera mitad de $1635^{10}$.

En su prólogo, Castillo no esboza ninguna conexión entre los materiales poéticos y aquellos otros en prosa, prefiriendo utilizar los versos como escudo contra los zoilos que podrían criticar sus prendas de historiador ${ }^{11}$. Tampoco explora la combinación de las formas en las observaciones que preceden o suceden a dichas composiciones: se limita a acompañarlas con la alabanza del «ingenio» de los respectivos autores, siendo el pobre Cruyllas el único a quien le escatima algún epíteto de este jaez. Sin embargo, la relación entre la poesía y la historiografía, y lo que podrían tener en común, es un tema que sí había suscitado algunos comentarios en las letras del Siglo de Oro. Por ejemplo, al escrutar ambas formas

10 Llama la atención la calidad de los receptores de sus dedicatorias en este periodo; por ejemplo, ofrece su Patrón de Alcira, texto valenciano a todos los efectos, a Baltasar Navarro Arroytia, obispo de Tarazona; dirige la Historia de Marco Antonio y Cleopatra a Juan de Moncayo, no solo marqués de San Felices, sino también poeta de cierto renombre en el ámbito regional; dedica el Epitome de la vida y hechos del ínclito rey don Pedro de Aragón a Antonio Jiménez de Urrea, conde de Aranda. La decisión de Castillo Solórzano de redactar esta historia, publicada en 1639 pero aprobada en 1636 y dedicada en forma manuscrita a Juan Funes de Villalpando, marqués de Osera, se debe entender también como una tentativa de granjearse simpatías en la región.

11 «Lector amigo, cuando por lo escrito de mi parte censures este breve volumen, habrás de abstenerte de mordaz, para con los grandes poetas que le honran con sus elegantes versos, padrinos de que me he valido, desconfiado de que en la disposición de la Historia habré faltado» (Castillo Solórzano 1639: «Prólogo»). 
genéricas en su tratado de 1611, De historia, para entenderla y escribirla, el teórico Luis Cabrera de Córdoba estableció una distancia conceptual entre la historia y la poesía, al tiempo que reconocía que pueden compartir objetivos. A su juicio, «la diferencia es [de] verdades, con que excluye la narración de la poesía, que es de mentiras; y así es mucha la diferencia y desconveniencia entre la historia y la poesía, no por ser esta en verso» (Cabrera de Córdoba 1948: 24). A su vez, contrapone la búsqueda de lo particular o real por parte del historiador al deseo de lo ideal o universal manifestado por el poeta (25). Empero, acepta la premisa según la cual la historia y la poesía comparten el objetivo de «condena[r] los vicios [y] alaba[r] las virtudes [...]. Ambas con suma industria atienden a la guardia de la prudencia y del decoro, enseñan, delectan, mueven, ayudan» (27). En este mismo sentido, la distinción tiene que ver más con su orígenes o naturalezas que con una separación absoluta entre ambas: «La historia tiene más de lo honesto, grave, ejemplar, como matrona ilustre y sabia. La poesía [de lo] joven y gentil, alguna vez con gala y hermosura» (28).

En la Historia de Marco Antonio y Cleopatra, la poesía puede ser de dos tipos: realza un episodio presentado ya en la prosa, resumiéndolo, recapitulándolo o ampliándolo; o bien subraya el mensaje moral, pero sin sustituir en ningún caso a una sección en prosa. Como decimos, los poemas son en efecto interpolados, en lugar de resultar básicos o constitutivos del volumen. La naturaleza recapitulativa de la mayoría de las composiciones mitiga la primera diferencia destacada por Cabrera, aquella entre la verdad de la forma prosística y las «mentiras» de la poética, mientras que los poemas morales aumentan el tono ejemplar de la historia y confirman la validez de lo declarado por el preceptista sobre el didactismo de tales géneros.

Por ejemplo, el soneto de Villanova, «Esta que así alentada se resiste» (Castillo Solórzano 1639: 133-134), composición con rasgos cultos y conceptistas, conmemora con la «gala y hermosura» imaginada por Cabrera la tumba de los amantes epónimos, introducida ya en la prosa del párrafo precedente; mientras que, a propósito del soneto de Montalbán relativo a la muerte de Pompeyo, Castillo enfatiza en su breve introducción el impacto moral generado por el texto (17). Otro poema con el mismo protagonista, el de Molina y Soto, «Para que el corazón más denodado» (11-12), abunda en los detalles de la huida de Farsalo, exhortando al lector en el primer cuarteto a que «pruebe la utilidad del escarmiento» inherente a la caída del general.

Si estas composiciones demuestran que los protagonistas titulares no dominan enteramente el volumen y que la carga ejemplar de la historia no recae únicamente sobre la prosa, es indudable que Cleopatra y Marco Antonio encarnan en mayor grado la ejemplaridad de los sucedidos, esencialmente en la prosa. En su dedicatoria, Castillo describe a los actores del libro como «varones ilustres», epíteto que engloba tanto los «hechos heroicos y honestos» como «los opuestos a 
ellos». Se debe emular la primera categoría y «huir de [la] dañosa imitación [de la segunda], con el escarmiento de sus violentos y desdichados fines». Si tal formulación incluiría también a Pompeyo y Julio César, las aseveraciones morales más explícitas se refieren al triunviro y a su amante, sobre todo al final de la narración:

Sirva de ejemplo la pérdida de Antonio para que los hombres no se cieguen en sus pasiones y libidinosos apetitos, pues este era un valeroso y fuerte capitán, experimentado en cualquiera trance... Y olvidado de sus obligaciones, hechizado con la hermosura de una libre reina de Egipto, fue vencido de su contrario, aborrecido del Senado, dado por enemigo de la patria, y últimamente muerto desesperadamente... Sirva asimismo de ejemplo, para que las mujeres se guarden, la libertad y desenvoltura de Cleopatra, el demasiado cuidado en aderezarse y componerse, porque las madres (a cuyo cargo está la crianza de sus hijos) les eviten esto cuando es con demasiado exceso; pues, de usarlo para parecer bien a los hombres, suceden mil desgracias y afrentas por casas y familias ilustres, con que vienen a menoscabo su fama (137-138).

La ejemplaridad de la Historia de Marco Antonio y Cleopatra, claro está, es aplicable a todos los estados de los hombres, no solo a los personajes ilustres; y en esto concuerda Castillo con otra historia -mucho más ambiciosa- basada en paradigmas romanos que influyeron en los comportamientos de la Edad Moderna. En el prólogo de su Historia imperial, citada ya en la primera parte de nuestro estudio, Mexía postulaba la historia como el recurso intelectual más importante para los que se hallan compelidos a gobernar, subrayando en paralelo la doble utilidad de la escritura histórica: si «los reyes y príncipes hallan en la historia otros a quien imiten y con quien compitan en virtudes y excelencias, y otros malos de cuyas costumbres huyan, y de cuyos fines y fama escarmienten», se debe reconocer igualmente que «este fruto y provecho es común a todo género de hombres» (Mexía 1655: «Al lector»). Esta concepción de la historiografía es muy común en el Siglo de Oro, pero nuestra cita de Mexía no es nada baladí, habida cuenta de que Castillo alude explícitamente a este texto al final de la Historia de Marco Antonio y Cleopatra; justo cuando esboza las fuentes a las cuales recurrió para escribirla:

Los autores que escriben todo lo que se ha dicho en este breve volumen son Tito Livio; Cornelio Tácito, en su primer libro; Plutarco, en la Vida de Julio César; Josefo, en el libro décimo de las Antigüedades; Lucano, en el primero y segundo libro; Pineda, y Pedro Mexía, en sus Césares (150) ${ }^{12}$.

12 Para un resumen de la historiografía clásica relativa a la vida de Cleopatra, véase Volkmann 1958: 220-228. Williamson (1974: 2; 26-31) anotó que los tratamientos históricos de Cleopatra que dominan en el Renacimiento son distintos de los del Medioevo; en particular, el de Plutarco 
A primera vista, este rosario de nombres parece equivaler a una confesión sincera de sus modelos. Huelga subrayar que Castillo, con tamañas autoridades, confería una solidez categórica a su relato, amén de eliminar hasta cierto punto -a diferencia de lo que ocurría con la lírica intercalada- cualquier titubeo sobre la adquisición de los materiales en prosa. Y si es verdad que el cuerpo de la historia no abunda en reconocimientos explícitos, a los númenes del listado antedicho se pueden añadir los de Casio Dion, Suetonio, Orosio, Plinio el Viejo, Ovidio, Ateneo y Galeno, a quienes el autor del Trapaza cita con diversos grados de especificidad. Sin embargo, la aparente franqueza de Castillo al final del libro oculta su auténtico modus operandi a la hora de construir el relato. Del par de cronistas españoles modernos, prefiere con mucho la Historia imperial de Mexía a la enciclopédica Monarquía eclesiástica de Pineda. De ahí que solo acudiera dos veces a esta última en la Historia de Marco Antonio y Cleopatra. Nótese además que dichos excerpta proceden de la misma parte del libro de Pineda (1588: f. 302v), tomado aquí como intermediario para reciclar un detalle de las Antigüedades judías de Josefo sobre el pérfido anhelo del poder absoluto por parte de Cleopatra (Castillo Solórzano 1639: 72), y para citar luego a Galeno a propósito de un manual apócrifo que habría escrito la reina acerca de los cosméticos (121-122). La historia de Mexía, por el contrario, facilita al vallisoletano la mayoría del material sobre César y la creación del Segundo Triunvirato, sin orillar las intrigas de Octaviano tras la muerte de Antonio y Cleopatra, a medida que este último avanzaba hacia su destino como César Augusto. Esto significa que el contenido que Castillo recicló de Mexía rodea la narración central de los amantes titulares, a la cual lógicamente no puede contribuir en demasía la Historia imperial del sevillano ${ }^{13}$.

La extrema dependencia de Castillo - respecto a otro escritor- por lo que concierne a una porción de su historia -no solo el contenido, sino las oraciones ad pedem litterae - apenas extraña en una época en la que la noción de la propiedad intelectual es manifiestamente más tenue que en la nuestra. Baste señalar, pues, que la gran modificación efectuada por Castillo se cifra en la compresión del texto original o en la omisión de detalles para dar pie a una narración menos morosa. Lo que podría despistar al lector, empero, es su declaración-dentro del epítome-de que ha consultado a «Plutarco en la Vida de Julio César». Podemos afirmar sin ningún género de duda que no hay en la Historia de Marco Antonio y Cleopatra nada del texto del biógrafo y moralista griego. Para decirlo de una vez, todas las supuestas citas de Plutarco en la primera sección de la historia o provienen del

(sobre todo en su Vida de Marco Antonio) sustituye a los más sencillos y moralizadores de los romanos Patérculo y Floro.

13 Castillo Solórzano (1639: 6-45) sigue -no siempre de modo literal- a Mexía (1655: 8-22), que trataba primero sobre Julio César (8-18) y luego sobre Octaviano. Véanse Castillo Solórzano (1639: 135-149) y Mexía (1655: 30-34). 
texto de Mexía (para quien Plutarco sí que representa una fuente primaria), o se introducen artificialmente en momentos claves para promover la impresión falsa de una lectura del sabio de Queronea. Solo cuando Mexía (1655: 22) se limita a resumir la estancia de Antonio en Egipto, abocetada tal que así: «Marco Antonio se dio a vicios y deleites después en Egipto con la reina Cleopatra», la prosa de Castillo debe alejarse necesariamente del texto del sevillano, uniéndose a su segunda fuente principal, la cual le suministró los hitos del triunviro y la reina: la Vida de Marco Antonio del citado Plutarco, esta vez sí adaptada por Castillo, aunque no lo reconozca apenas, desde el vigésimo quinto capítulo hasta el final ${ }^{14}$.

A la luz del abusivo uso de esta otra Vida, hay que preguntarse por qué Castillo confiesa, de manera falaz, que ha acudido a la Vida de Julio César, pasando muy de puntillas sobre sus deudas con la de Marco Antonio. Una explicación plausible sería que Castillo quiere esconder su dependencia, casi absoluta, de una sola fuente moderna, la de Mexía, para redactar la sección primera de la Historia de Marco Antonio y Cleopatra. Tal ofuscación se ve reforzada por dos falsedades subsidiarias, ya que las alusiones a Livio y Lucano en la lista terminal corresponden a citas en la primera parte del texto de Castillo que no procedían de un cotejo directo de las autoridades clásicas, sino, una vez más, de la mediación de la obra de Mexía. Y la segunda causa es que Castillo despliega su ardid a propósito de su frecuentación de la Vida de Julio César para distraer al lector de los préstamos, más sustanciales y no confesos, de la Vida de Marco Antonio del mismo Plutarco.

Desde otra ladera, Castillo hace suyos algunos episodios de otras autoridades en esta parte central de la Historia de Marco Antonio y Cleopatra-hemos aducido ya los casos de Josefo y Galeno, extraídos de la Monarquía eclesiástica de Pineda, y la descripción del primer banquete que Cleopatra ofrece a su amante, próximo al de los Deipnosophistae de Ateneo, quizá filtrado a través de algún florilegio-. No obstante, la segunda fuente a la que recurre ahora el vallisoletano es la Historia romana de Casio Dion, criticado severamente por Cabrera a causa de su reducida fiabilidad ${ }^{15}$. Esta alianza de las obras de Plutarco y Dion -a la postre decantada en favor del primero- se menciona una sola vez y en términos muy vagos: «Muchos errores hizo Antonio, siendo valeroso capitán y de mucha experiencia, los cuales pudiera remediar y ser vencedor, como se verá en Plutarco y en Dion, que

14 El texto de Castillo Solórzano (1639: 45) corresponde al capítulo 25.1 de la Vida de Marco Antonio, y la historia de Castillo sigue a Plutarco hasta el capítulo 87.2 (véase Castillo Solórzano 1639: 135). El texto griego se puede consultar en Plutarco (1988: 49-115).

15 Para Cabrera, Dion es uno de los escritores que «mienten entre los griegos [...] en las cosas de Asiria, Egipto y Fenicia» y un historiador que «en las antigüedades romanas falt[a] a la verdad» (Cabrera de Córdoba 1948: 45, 100). Mientras Cabrera no considera infalible a Plutarco -según Cabrera tiene una tendencia a escribir de manera demasiado descriptiva (81)-, lo presenta como un autor que escribió con precisión de los asuntos de la Grecia y la Roma antiguas $(45 ; 60)$ y que sabe representar los episodios lascivos de manera decorosa (84). 
escriben su vida» (Castillo Solórzano 1639: 87) ${ }^{16}$. Pero es justo esta aleación la que confiere al libro de Castillo su mayor fuste historiográfico: el autor del Trapaza entreteje los textos con referencia no a sus originales griegos, ni siquiera a sus versiones en latín, sino por medio de las traducciones en italiano rubricadas por Lodovico Domenichi (1555) y Francesco Baldelli (1565) respectivamente, y del relato extenso, y por lo general creíble, de Plutarco, que le sirve para vertebrar la trama de los amantes. Cierto asimismo que Castillo agrega detalles y episodios derivados de Dion que aumentan o sustituyen a las partes correspondientes en Plutarco ${ }^{17}$. Si los poemas constituían el ingrediente más llamativo y novedoso del collage de esta historia mixta, la hibridación -hasta ahora inadvertida- de las obras de Plutarco y Dion representa su faceta más sofisticada y ejemplarizante.

Para ilustrar esta dinámica, vamos a reparar en uno de los lances más densos: las desesperadas maniobras de Antonio y Cleopatra después de la Batalla de Accio (Castillo Solórzano 1639: 99-104). La descripción de los experimentos que ensaya Cleopatra con varios venenos animales -antes de decidirse por el del áspid- deriva de Plutarco (1555: 366), mientras que las tres opciones que abren el capítulo siguiente - una fuga en España, donde pervive la rebelión contra Octaviano; escapar por el mar Rojo; o, la tentativa más vil, ordenar el asesinato de Octaviano- provienen de Dion (1565: 553). La arriesgada propuesta de Cleopatra, según la cual Octaviano permitiría a Marco Antonio exiliarse en Atenas y a los hijos de ella gobernar en Egipto, está tomada de Plutarco (1555: 366), pero la perfidia de la reina, subrayada más tarde por Castillo, falta en el texto del queroneo, y no así en el de Dion:

Y Cleopatra, de secreto (sin que Antonio fuese sabedor de esto), envió a César un cetro y una corona de oro, y la Silla Real de los reyes de Egipto, que era pieza riquísima y todas tres de inestimable valor, por sobornarle con estos dones, para que disimuladamente le entregase a ella el reino; y que si la enemistad era con Antonio, de ella, por lo menos, tuviese misericordia $(102-103)^{18}$.

Castillo sigue la falsilla de Dion (1565: 553) al describir la reacción de Octaviano, pero los pormenores del viaje de Tirso a Alejandría, enviado por Octaviano,

16 Las partes de la Historia romana que utiliza Castillo se hallan entre 49.40 y 51.15 . El texto griego se puede consultar en Dion (1917a: 422-515) y Dion (1917b: 1-44).

17 Castillo saca sus materiales de Plutarco (1555: 340-373) y Dion (1565: 513-561). La glosa muy detallada que Pelling aporta a la Vida de Marco Antonio (Plutarco 1988: 117-327) identifica los puntos en común entre la versión del sabio de Queronea y las de otros historiadores clásicos, señalando también los puntos en los que divergen, sobre todo respecto a la versión de Dion.

18 Castillo sigue muy de cerca la traducción de Baldelli: «Cleopatra intanto mandó di nascoso, sí che Antonio non ne seppe nulla, il scettro d'oro, la corona d'oro, e la Sedia Reale a Cesare, quasi che, dandogli queste cose, gli concedesse con esse il regno, accioché s'egli portasse tutto l'odio suo ad Antonio, ella potesse almeno ottenere misericordia da lui» (Dion 1565: 553). 
provienen de Plutarco (1555: 367), pese a que una versión análoga se registra también en Dion (1565: 555). Cuando, poco después, Octaviano se impone en Pelusio, Castillo trae la tesis desarrollada por Dion (1565: 556), para quien la traición a la ciudad por parte de Cleopatra y su amante no es una posibilidad, como había sugerido Plutarco, sino un hecho incontrovertible, por más que luego añada un dato extraído de Plutarco (1555: 367): Cleopatra inculpó a Seleuco y entregó a su mujer e hijos a Antonio para que este se vengara de ellos.

$\mathrm{Al}$ entretejer sus dos fuentes, Castillo apuesta por presentar una versión de los hechos que a cada paso nos devuelve un retrato poco favorable de Cleopatra. Pelling (1995: 149) explicó que el primer tercio de la Vida de Marco Antonio de Plutarco ostenta un tono moralizador, mientras que esta faceta disminuye notablemente cuando el historiador introduce a la reina: «The story is immediately seized by a new narrative and descriptive vigour ... There are no more intrusive moralizing remarks; no more explicit denunciations of the actions he describes ... Praise and blame are alike irrelevant to the narrative». Pelling subraya también que Plutarco evalúa la infatuación de Marco Antonio y sus desastrados efectos con «psychological empathy», y, en comparación con otros historiadores, pinta un «portrait of greater humanity ... [and] deserves credit for such imaginative compassion» (Plutarco 1988: 18). Por tanto, Castillo asimiló la profundidad narrativa y la compleja caracterización del texto de Plutarco para diseñar una historia amena y convincente, aun cuando la tuerza en aras de un objetivo moral bien distinto. En paralelo, el vallisoletano acude a Dion para deslizar una ejemplaridad negativa ajena a la de Plutarco, cifrada a su vez en la escena crucial de la entrevista de Cleopatra con Octaviano tras el suicidio de Antonio (Castillo Solórzano 1639: 121-124): Castillo no solo convierte a Cleopatra en protagonista activa -es ella quien organiza el encuentro, y no el triunviro- y modifica la sede del encuentro del mausoleo al palacio de la soberana -ambas anécdotas recicladas de Dion (1565: 558-559)-, sino que sustituye enteramente a la mujer emocional y físicamente venida a menos que nos presentara Plutarco ${ }^{19}$ por una hábil manipuladora que se valdrá de la belleza y el chantaje sentimental -mediante la evocación de su antiguo amor, Julio César, padre adoptivo de Octaviano- para atraerse la simpatía de Octaviano. En este punto, Castillo vuelve a aprovecharse de la obra de Dion:

Sabido esto por Cleopatra, mandó aderezar su casa con las más ricas y costosas colgaduras que tenía [...] El vestido con que Cleopatra recibió esta visita fue de luto, que este hábito le estaba más bien, aunque con cualquiera parecía siempre hermosa

19 En la traducción de Domenichi, se lee que Cleopatra, «come lo vide venire [a Marco Antonio], gettatasi fuor del letto, dov'ella umilmente giaceva, solamente con la prima veste indosso, si gl'inginocchiò a piedi, con voce debole e tremante [...] Né in cosa alcuna pareva che'l corpo stesse punto meglio che l'anima» (Plutarco 1555: 371). 
[...]. Para aguardar esta visita se sentó en una silla, y en torno de ella puso cerca de sí muchos retratos del primero César. Y asimismo tenía consigo muchas cartas que de este había recibido, y copias y borradores de otras que ella le había escrito estando ausente (Castillo Solórzano 1639: 121-122) ${ }^{20}$.

De acuerdo con lo dicho hasta aquí, la Historia de Marco Antonio y Cleopatra es el depósito literario más nutrido de materiales sobre Cleopatra a lo largo del Siglo de Oro. Incluso en su versión primitiva, ya hubiera supuesto una contribución capital a la imagen protomoderna de la reina egipcia; de modo que la adición de casi una treintena de poemas solo podía incrementar esta jerarquía. El libro que salió de las prensas de Vergés en 1639 confirma a Castillo como uno de los exponentes más destacados de la escritura mixta, y convierte en aún más proteico el periodo más variado de su carrera. Como historia popular es entretenida y plausible, y como antología poética no solo preserva composiciones de personajes de buenas prendas cuya producción impresa se antoja muy reducida, verbigracia la de Francisco Diego de Sayas, sino que ilustra también el carácter liminar de las prácticas compositivas entre los escritores profesionales y los meros diletantes.

Los poemas son aquí bastante más que decoraciones a vuela pluma que confieren «gala y hermosura» al volumen. Se insertan en el texto para rectificar las deficiencias del neófito historiador que era Castillo, resaltando de paso episodios claves de la tumultuosa historia, a menudo apuntalada por la moralidad de la prosa. Castillo reconoce el poder y la seducción de atributos como la lujuria y la avidez, encarnados en la seductora Cleopatra, pero los condena en términos directos e inequívocos, o bien -y nos parece más sugestivo- injertando le negatividad de Dion en el palimpsesto histórico de Plutarco. Aquí estriba la tesela oculta del collage de la Historia de Marco Antonio y Cleopatra, la misma que desmiente la falsa modestia del autor vallisoletano cuando en su prólogo desconfiaba de que «en la disposición de la Historia habr[ía] faltado».

Corresponde a Dión 1565: 558: «Cleopatra allora avendo apprestata una bellissima stanza con ricchissimi ornamenti, e una richissima sedia, ornò la persona sua con una maniera d'ornato quanto più negletto fosse possibile (conciófossecosache quell'abito lugubre la facesse a riguardare maravigliosa) e misesi a sedere nella seggiola, havendo fatto attaccare intorno a sé molti e molti ritratti dell'altro Cesare, e avevasi messo in seno le lettere da esso scrittele». 


\section{BIBLIOGRAFÍA CITADA}

ARREDONDO, María Soledad (2006). «Castillo Solórzano y la mixtura barroca: poesía, narrativa y teatro en La niña de los embustes, Teresa de Manzanares». En Odette Gorsse y Frédéric Serralta (coords.), El Siglo de Oro en escena. Homenaje a Marc Vitse. Tolosa: Presses Universitaires du Mirail, pp. 35-51.

Boaistuau, Pierre (1586). Historias prodigiosas y maravillosas de diversos sucessos acaescidos en el mundo. Andrea Pescioni (trad.). Medina del Campo: Francisco del Canto.

Bonilla Cerezo, Rafael (2012). «Alonso de Castillo Solórzano: bio-bibliografía completa». Tintas. Quaderni di Letterature Iberiche e Iberoamericane, 2, pp. 243-282.

BorJa, Francisco de (1648). Obras en verso. Madrid: Diego Díaz de la Carrera.

Cabrera de Córdoba, Luis (1948). De historia, para entenderla y escribirla. Santiago Montero Díaz (ed.). Madrid: Instituto de Estudios Políticos.

Castillo Solórzano, Alonso de (1634). Fiestas del jardín. Valencia: Silvestre Esparsa. (1639). Historia de Marco Antonio y Cleopatra. Zaragoza: Pedro Vergés.

(1907). Tiempo de regocijo y Carnestolendas de Madrid. Emilio Cotarelo y Mori (ed.). Colección selecta de antiguas novelas, vol. 2. Madrid: E. Maestre.

(1944). Huerta de Valencia. Prosas y versos en las academias de ella. Madrid: Sociedad de Bibliófilos Españoles.

Dion, Casio (1565). De'fatti de' romani. Francesco Baldelli (trad.). Venecia: Gabriele Giolito.

(1917a). Roman History, vol. 5. Earnest Cary (ed. y trad.). Cambridge, Massachusetts: Harvard University Press.

(1917b). Roman History, vol. 6. Earnest Cary (ed. y trad.). Cambridge, Massachusetts: Harvard University Press.

Fuentes, Alonso de (1587). Libro de los cuarenta cantos. Alcalá de Henares: Juan Gracián.

GonZÁlez CAÑAL, Rafael (2008). «Cleopatra, una figura femenina del teatro de Rojas». En Felipe B. Pedraza Jiménez, Rafael González Cañal y Elena E. Marcello (coords.), Rojas Zorrilla en su IV centenario. Cuenca: Universidad de Castilla-La Mancha, pp. 269-291.

JÁuREGUI, Juan de (1618). Rimas. Sevilla: Francisco de Lyra Varreto.

JimÉNEZ BELMONTE, Javier (2011a). «Historiar el Oriente: Cleopatra en la historiografía española del XVI». eHumanista, 17, pp. 286-310.

(2011b). «De Cleopatra y mecenazgos: la Historia de Marco Antonio y Cleopatra de Alonso de Castillo Solórzano (Zaragoza, 1639)». En Antonio Azaustre Galiana y Santiago Fernández Mosquera (coords.), Compostella Aurea. Actas del VIII Congreso de la AISO, vol. 2. Santiago de Compostela: Universidade de Santiago de Compostela, pp. 315-322.

KING, Willard F. (1963). Prosa novelística y academias literarias en el siglo XVII. Madrid: Real Academia Española.

Mas i Usó, Pasqual (1999). Academias valencianas del Barroco. Descripción y diccionario de poetas. Kassel: Reichenberger.

MexíA, Pedro (1655). Historia imperial y cesárea. Madrid: Melchor Sánchez. (2003). Silva de varia lección. Isaías Lerner (ed.). Madrid: Castalia. (2004). Diálogos o Coloquios. Antonio Castro Díaz (ed.). Madrid: Cátedra. 
Pelling, C.B.R. (1995). «Plutarch's Adaptation of his Source-Material». En Barbara Scardigli (coord.), Essays on Plutarch's «Lives». Oxford: Oxford University Press, pp. 125154.

PINEDA, Juan de (1588). Los treinta libros de la Monarquía eclesiástica, o Historia universal del mundo, vol. 1. Salamanca: Juan Fernández. (1963). Diálogos familiares de la agricultura cristiana, vol. 1. Juan Meseguer Fernández (ed.). Madrid: Atlas.

Plutarco (1555). La seconda parte delle Vite. Lodovico Domenichi (trad.). Venecia: Gabriele Giolito.

(1988). Life of Antony. C. B. R. Pelling (ed.). Cambridge: Cambridge University Press.

Rennert, Hugo (1908). «Marco Antonio y Cleopatra. A Tragedy by Diego López de Castro». Revue Hispanique, 19, pp. 184-237.

SAINZ DE LA MAZA, Carlos (2009). «El banquete de Cleopatra, I: los textos y sus antecedentes». En Esther Borrego Gutiérrez y Catalina Buezo Canalejo (coords.), Literatura, política y fiesta en el Madrid de los Siglos de Oro. Madrid: Visor, pp. 421-451.

VeGA, Lope de (1973). El peregrino en su patria. Juan Bautista Avalle-Arce (ed.). Madrid: Castalia.

Volkmann, Hans (1958). Cleopatra. A Study in Politics and Propaganda. T. J. Cadoux (trad.). Londres: Elek.

Williamson, Marilyn L. (1974). Infinite Variety: Antony and Cleopatra in Renaissance Drama and Earlier Tradition. Mystic, Connecticut: Lawrence Verry.

Recibido: $15 / 7 / 2016$

Aceptado: 3/11/2016 


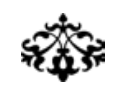

\section{Un COLLAGE DE FUENTES EN la Historia DE MARCo ANTONio y Cleopatra (1639) de Castillo Solórzano}

Resumen: La Historia de Marco Antonio y Cleopatra (1639) de Castillo Solórzano es el depósito literario más nutrido de materiales acerca de Cleopatra a lo largo del Siglo de Oro. Se trata de un relato popular que entretiene mientras promueve una clara visión ejemplar: Castillo reconoce el poder y el atractivo de atributos como la lujuria y la avidez, al tiempo que las condena por medio de Cleopatra, Marco Antonio, Julio César, Pompeyo y Octaviano. Lo que confiere singularidad a esta historia en prosa es la inclusión de casi treinta poemas, del mismo Castillo y de un rosario de poetas de Valencia, Madrid y Zaragoza; un detalle que confirma al vallisoletano como uno de los exponentes más señeros de la escritura mixta durante el Barroco. Este estudio analiza el carácter de los materiales en prosa y en verso, considerando la obra como un collage de fuentes. El examen de la aleación de estos modelos -visibles u ocultos- y de sus grados de integración pone de manifiesto los objetivos artísticos y morales del autor.

Palabras Clave: Alonso de Castillo Solórzano, Historia de Marco Antonio y Cleopatra, Plutarco, Casio Dion, fuentes, literatura mixta.

\section{A Collage of Sources in Castillo Solórzano's Historia de Marco Antonio y Cleopatra (1639)}

AbSTRACT: Alonso de Castillo Solórzano's Historia de Marco Antonio y Cleopatra (1639) is the most important literary repository for materials on Cleopatra in the whole of the Spanish Golden Age, and it is a popular history which entertains while also promoting a clear moral vision. Castillo acknowledges the power and attraction of characteristics such as lust and greed, but at the same time he condemns them through the characters of Cleopatra, Antony, Julius Caesar, Pompey and Octavian. What bestows special particularity upon this prose history is the inclusion of almost thirty poems, by Castillo himself and by poets from Valencia, Madrid and Zaragoza, and this confirms Castillo as one of the foremost exponents of mixed writing in the Baroque. This study analyses the character of the two types of material, prose and verses, and considers the work as a collage of sources. The examination of the way in which these sources -visible or hidden-combine, and of their degrees of integration makes clear Castillo's moral and artistic aims.

Keywords: Alonso de Castillo Solórzano, Historia de Marco Antonio y Cleopatra, Plutarch, Cassius Dio, sources, mixed writing. 
Alonso de Castillo Solórzano (1584-¿1648?)

PaOlo Pintacuda (Università degli Studi di Pavia)

La tradición impresa de los Donaires del Parnaso (1624-1625) ............................... 11

Rocío Jodar Jurado (Universidad de Córdoba)

El collar de Adonis: Castillo Solórzano, Diego de Frías

y Miguel de Barrios en la estela de Ovidio.

Marcial Rubio Árquez (Università degli Studi G. D’Annunzio di Chieti-Pescara)

Huerta de Valencia: entre la colección de novelas

y el cancionero de academia.

Cristina Castillo Martínez (Universidad de Jaén)

La escritura hagiográfica de Castillo Solórzano.

JAUME GARAU (Universidad de las Islas Baleares)

El tratamiento de la hagiografia en Castillo Solórzano

a la luz de la Contrarreforma

JoNATHAN BRADBURY (University of Exeter)

Un collage de fuentes en la Historia de Marco Antonio y Cleopatra (1839)

de Castillo Solórzano.

LuANA BeRmúDEZ (Université de Genève)

Las harpías en Madrid y coche de las estafas, de Castillo Solórzano:

entre novela y puesta en escena

Belinda Palacios (Université de Genève)

Alegoría y comicidad en el auto sacramental de Alonso de Castillo Solórzano

El fuego dado del cielo

\section{OTROS TEMAS}

Josep Lluís Martos (Universidad de Alicante)

La fecha del Cancionero de romances sin año.

María Gabriela Huidobro Salazar (Universidad Andrés Bello)

Recursos literarios de la épica clásica para la representación

de la Guerra de Arauco en el siglo XVI.

Ana Aparecida Teixeira de Souza (Universidade de São Paulo)

La representación de la fortuna y los cambios de identidad

en El laberinto de amor de Miguel de Cervantes. 


\section{EDAD DE ORO}

Revista de Filología Hispánica XXXVI

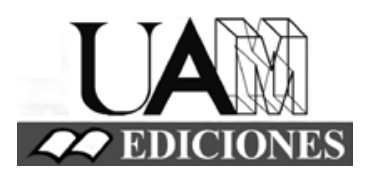




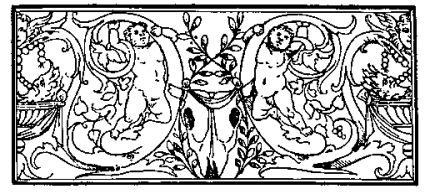

\section{Edad de Oro. Revista de Filología Hispánica}

ISSN: 0212-0429

Dirección:

Teodosio Fernández

Secretaría y edición:

José Ramón Trujillo

Consejo de redacción:

Manuel Piqueras

Blanca Santos

Admisión de originales:

María Jesús Zamora

Edad de Oro

Departamento de Filología Española

Universidad Autónoma de Madrid

28049 Madrid (España)

Tfno.: +0034914974090

correo: mariajesus.zamora@uam.es

Distribución, suscripción y venta:

Servicio de Publicaciones de la UAM

Universidad Autónoma de Madrid

28049 Madrid (España)

Intercambio de publicaciones:

Biblioteca de la Facultad de Filosofía y

Letras (UAM)

Universidad Autónoma de Madrid

28049 Madrid (España)
Comité científico internacional:

Carlos Alvar (Univ. de Ginebra)

Ignacio Arellano (Univ. de Navarra)

Javier Blasco (Univ. de Valladolid)

Alberto Blecua (UAB)

Jean Canavaggio (Univ. de París X)

Laura Dolfi (Univ. de Turín)

Aurora Egido (Univ. de Zaragoza)

Víctor García de la Concha (RAE)

Luciano García Lorenzo (CSIC)

Joaquín González Cuenca (Univ. de

Castilla-La Mancha)

Agustín de La Granja (Univ. de Granada)

Begoña López Bueno (Univ. de Sevilla)

Michel Moner (Univ. de Toulouse III)

Joan Oleza (Univ. de Valencia)

Alfonso Rey (Univ. de Santiago)

Lina Rodríguez Cacho (Univ. de Salamanca)

Leonardo Romero Tobar (Univ. de Zaragoza)

Aldo Ruffinatto (Univ. de Turín)

Lía Schwartz (City University of New York)

Han colaborado en este volumen:

Departamento de Filología Española (UAM)

Facultad de Filosofía y Letras (UAM)

Edad de Oro se recoge, entre otras, en las siguientes bases de datos: SCOPUS, MLA Database, HLAS, Latindex, PIO-Periodical Content Index, ISOC, Dialnet, MIAR, ERIH Plus, DICE, Sumaris CBUC, Ulrich's. Se encuentra evaluada en CIRC: A; MIAR difusión ICDS live 2016: 10.0; INRECH; SCImago Journal \& Country Rank: H Index 3, SJR SCImago Journal \& Country Rank 0,1, Q4; RESH índice de impacto: 0.041; ERIH: A INT1; Carhus Plus+2014: C. 